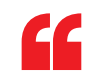

We expected longer acenes to show similar electrochemistry with different colour luminescence, but until now we haven't been able to make them

POLYCYCLIC AROMATIC HYDROCARBONS

\title{
Twisted targets
}

After a little over 20 years of trying, a team of researchers led by Robert Pascal from Tulane University have been able to prepare dodecaphenyltetracene. Writing in Angewandte Chemie, they describe the synthesis and characterization of this interesting twisted hydrocarbon.

The steric crowding from the 'picket fence' of phenyl rings at the periphery of the molecule (image, blue) twists the acene core (image, red), such that the first and fourth arene rings are roughly perpendicular $\left(97^{\circ}\right)$. "We (and others) have targeted these molecules largely for the synthetic challenge," says Pascal, "but they do have some unusual and interesting electrochemical properties."

The picket fence of substituents around the acene not only twists
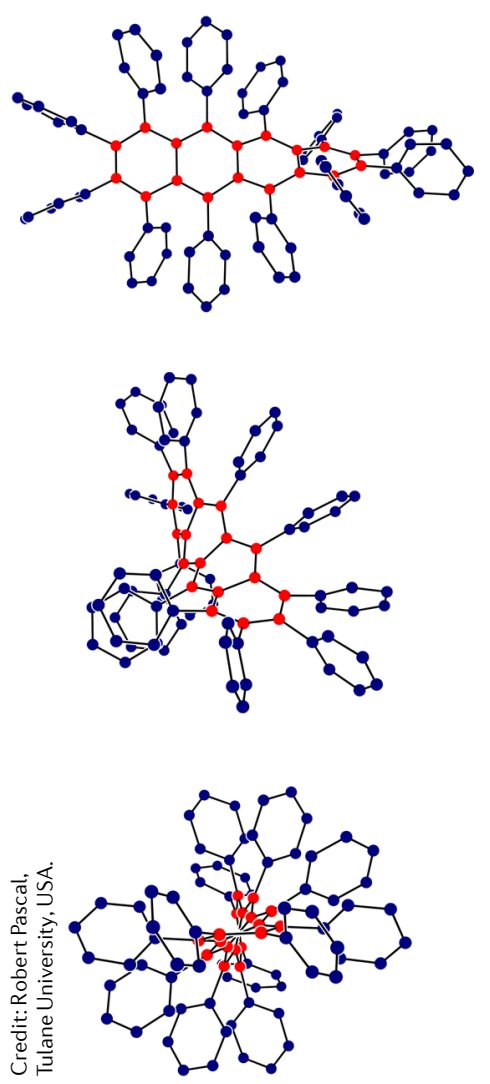

the core but also protects it and its ions, as evidenced by its fully reversible electrochemical oxidation and reduction waves. This redox stability makes species like dodecaphenyltetracene ideal for use in electrochemiluminescence, which is attractive in a number of analytical applications owing to a low background signal and easy control of the light-generating reaction.

"We made decaphenylanthracene in 1996," says Pascal. "It showed fully reversible electrochemical oxidation and yellow-green luminescence. We expected longer acenes to show similar electrochemistry with different colour luminescence, but until now we haven't been able to make them."

The reported synthesis of dodecaphenyltetracene uses iterative Diels-Alder reactions between benzyne and furan intermediates to construct the multiple six-membered rings of the acene. The yields of these reactions diminish when using longer acenes, presumably as a result of increased steric strain. The initial products are epoxyacenes - oxygen-bridged ring species whose reduction ultimately gives the acene.

"There's no one breakthrough that makes the synthesis work in this case," says Pascal. "Our success is the result of a careful re-examination of this benzyne approach by my student Yonglong Xiao. Even then, when we tried to prepare the larger analogue tetradecaphenylpentacene, the benzyne Diels-Alder reaction was successful, but reduction of the resulting epoxyacene has, so far, failed."

Despite their success in finally preparing the tetracene, Pascal says that he feels an alternative approach pioneered by the Kilway group from the University of Missouri, Kansas City, may be helpful in accessing longer analogues.
The alternative approach still relies on the Diels-Alder reaction but involves reacting a quinone sequentially with two dienes. In this case the lack of two large substituents on the central quinone ring appears to relieve strain sufficiently to allow the reaction to proceed more easily. The fully substituted acene can then be produced by nucleophilic attack of phenyllithium on the carbonyls followed by reduction of the resulting alcohols.

"The quinone approach has proven superior for the synthesis of many benzannnulated analogues, and I wouldn't be surprised to see it applied to longer perphenylacenes in the future," says Pascal.

The twist in the structure of these acenes results in a chiral conformation. However, unlike helicenes - related polycyclic aromatic hydrocarbons - these structures are unlikely to be configurationally stable under normal conditions. In the acenes, racemization occurs through a multistep process and each step has only a small activation energy. In contrast, helicene racemization occurs through a single step with a high activation energy. "The only highly twisted acene that has so far been separated into its enantiomers was found to have a racemization half-life of around 9 hours," says Pascal. "That molecule was considerably more configurationally stable than the perphenylacenes, and our calculations suggest that these molecules have a half-life of less than a second."

Stephen G. Davey

ORIGINAL ARTICLE Xiao, Y. et al. Dodecaphenyltetracene. Angew. Chem. Int. Ed. https://doi.org/10.1002/anie.201812418 (2019) FURTHER READING Clevenger, R. G. et al. A superior synthesis of longitudinally twisted acenes. Chem. Eur. J. 24, 243-250 (2018). 